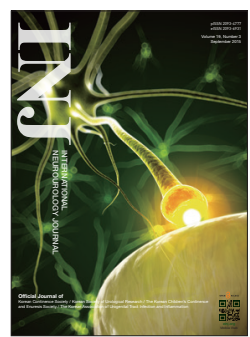

\title{
Implantable Bladder Sensors: A Methodological Review
}

\author{
Mathias Naangmenkpeong Dakurah ${ }^{1}$, Chiwan Koo $^{1}$, Wonseok Choi ${ }^{2}$, Yeun-Ho Joung ${ }^{1}$ \\ ${ }^{1}$ Department of Electronics and Control Engineering, Hanbat National University, Daejeon, Korea \\ ${ }^{2}$ Department of Electrical Engineering, Hanbat National University, Daejeon, Korea
}

\begin{abstract}
The loss of urinary bladder control/sensation, also known as urinary incontinence (UI), is a common clinical problem in autistic children, diabetics, and the elderly. UI not only causes discomfort for patients but may also lead to kidney failure, infections, and even death. The increase of bladder urine volume/pressure above normal ranges without sensation of UI patients necessitates the need for bladder sensors. Currently, a catheter-based sensor is introduced directly through the urethra into the bladder to measure pressure variations. Unfortunately, this method is inaccurate because measurement is affected by disturbances in catheter lines as well as delays in response time owing to the inertia of urine inside the bladder. Moreover, this technique can cause infection during prolonged use; hence, it is only suitable for short-term measurement. Development of discrete wireless implantable sensors to measure bladder volume/pressure would allow for long-term monitoring within the bladder, while maintaining the patient's quality of life. With the recent advances in microfabrication, the size of implantable bladder sensors has been significantly reduced. However, microfabricated sensors face hostility from the bladder environment and require surgical intervention for implantation inside the bladder. Here, we explore the various types of implantable bladder sensors and current efforts to solve issues like hermeticity, biocompatibility, drift, telemetry, power, and compatibility issues with popular imaging tools such as computed tomography and magnetic resonance imaging. We also discuss some possible improvements/emerging trends in the design of an implantable bladder sensor.
\end{abstract}

Keywords: Urinary Incontinence; Implantable Bladder Sensor; Hermetic; Biocompatible Materials; Telemetry

- Grant Support: The research was supported by the Ministry of Science, ICT and Future Planning through the Development for IT.SW industrial convergence original technology (ID: R0101-15-0147).

- Conflict of Interest: No potential conflict of interest relevant to this article was reported.

\section{INTRODUCTION}

Urinary incontinence (UI) is not only caused by peripheral nerve/spinal cord injury but also other factors such as excessive alcohol intake, pregnancy, childbirth, bladder tumor/cancer, and an enlarged prostate. As a result, every individual is at risk for urinary incontinence. In the United States alone, the estimated total national cost of UI in 2007 was $\$ 65.9$ billion, with projected costs of $\$ 76.2$ billion in 2015 and $\$ 82.6$ billion in 2020 [1]. Addi- tionally, according to the World Health Organization, roughly 20 million people are affected by UI annually, worldwide [2]

Over the years, measurement of abnormal bladder urine volume/pressure has been the key to restoring bladder function, creating awareness of when to empty the bladder, as well as improving the quality of life of patients with UI. In a clinical setting, it is common to see patients with UI carrying catheter-based bladder urine sensors around. Even though this method grants the accuracy needed for monitoring urine volume/pressure, it is

Corresponding author: Yeun-Ho Joung (iD http://orcid.org/0000-0003-2365-2805 Department of Electronics and Control Engineering, Hanbat National University, 125 Dongseo-daero, Yuseong-gu, Daejeon 34158, Korea E-mail: yeunho@gmail.com / Tel: +82-42-821-1166 / Fax: +82-42-821-1164 Submitted: August 5, 2015 / Accepted after revision: August 19, 2015 
invasive and provides limited, short-term information about the dynamic volume/pressure profile within the urinary bladder. Therefore, this method is suitable for hemiplegic patients and only feasible in clinical settings. To circumvent these problems, noninvasive monitoring methods using sound or electromagnetic radiation, such as light, ultrasound, and x-ray, have been studied to ensure efficient diagnosis and improve the efficacy of urinary bladder dysfunction therapy. However, these methods may require exposure of the body to high-energy waves that are not very accurate since they interfere with the pressure/volume of the urinary bladder as the tissues and fluids interact with these high-energy waves [3]. Therefore, fully implantable bladder sensors with telemetric mechanisms could provide direct measurement of bladder urine volume or pressure without the risks of infection posed by catheters, wires, or high-energy waves.

With the rapid advancement in micromachining/microfabrication over the years, miniaturized and highly accurate sensors are being developed that may safely be implanted into patients for long-term monitoring. Here, we explore the various types of implantable bladder sensors, design requirements and specifications, as well as current efforts to address issues including hermeticity, biocompatibility, drift, telemetry, power transfer, and compatibility issues with popular imaging tools such as computed tomography (CT) and magnetic resonance imaging (MRI). We also discuss some possible improvements/emerging trends in the design of implantable bladder sensors for enhanced patient comfort and long-term monitoring.

\section{TYPES OF IMPLANTABLE BLADDER SENSORS}

Over the last five decades, several techniques have been developed for the quantification of urinary bladder function. These techniques include indirect volume estimation from intravesical pressure, electrical impedance plethysmography, strain gauge plethysmography, imaging plethysmography using ultrasound sensors, electromagnetic plethysmography, and electroneurographic recordings from peripheral nerves [4]. In current clinical settings, catheterization and catheter-based sensors are the most common tools employed in the monitoring of urinary bladder volume. Catheter-based sensing is used as the reference standard for other sensors because of its accuracy and precision. However, this technique is very invasive and can cause infection during prolonged use. Therefore, it is only suitable for short-term usage and feasible only in a clinical milieu.

Earlier attempts at bladder urine volume sensing were performed by measuring the bioelectric impedance difference between electrodes sutured to the detrusor muscle on opposite sides of the bladder as one arm of a resistor-capacitor phase shift oscillator. This oscillator functioned as a transponder, transmitting the changes in wave frequency, as a result of bladder filling, to an external receiver. However, encapsulation of these electrodes by fibrotic tissue growth demanded the need for frequent calibration, thereby hindering its feasibility in clinical application [5]. To avoid biofouling, Dreher et al. [6] presented an alternative method of bladder urine volume sensing that used a reed switch
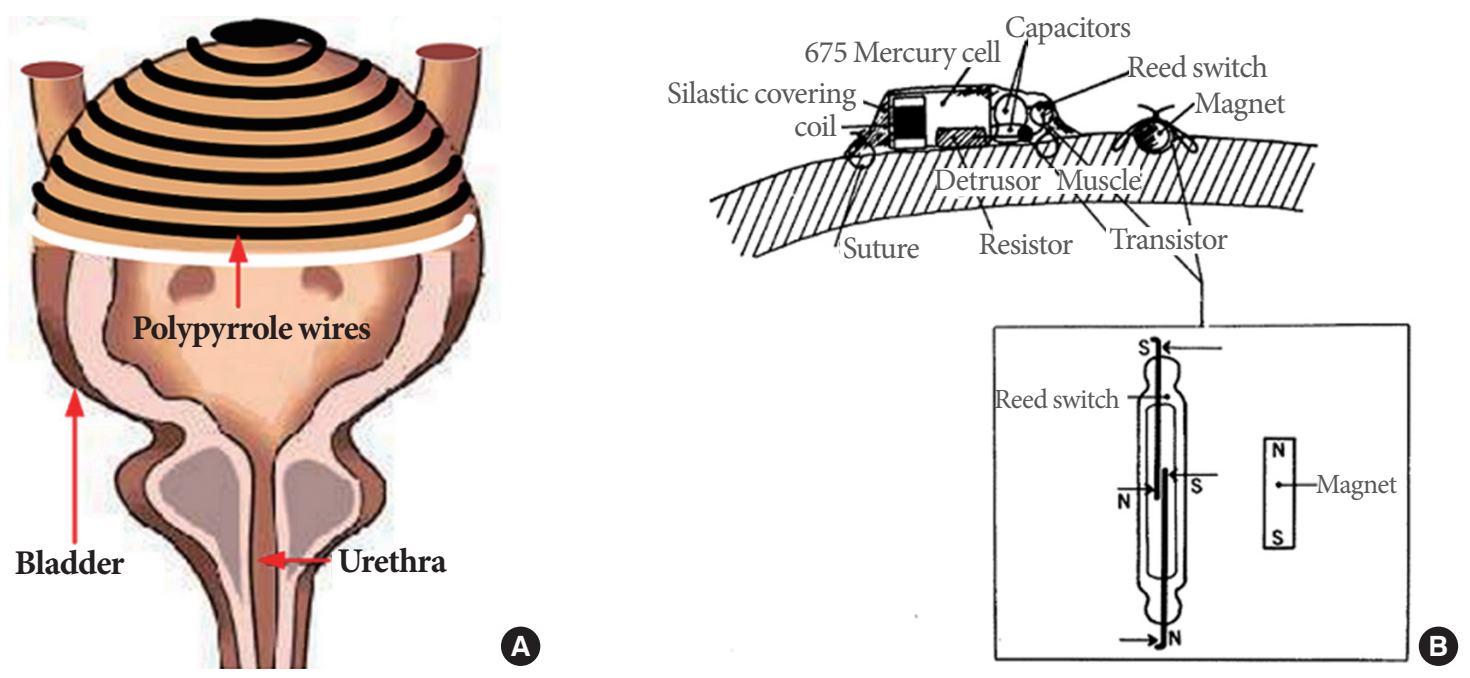

Fig. 1. Bladder volume sensing using a strain sensor (A: adapted from Rajagopalan S, et al. Sensors 2008;8:5081-95 [14], on the basis of Open Access) and a reed switch and magnet (B: adapted from Dreher RD, et al. IEEE Trans Biomed Eng 1972;19:247-8 [6], with permission of the Institute of Electrical and Electronics Engineers). 
and a magnet sutured to the detrusor muscle in close proximity to one another. As the urinary bladder volume rises, the distance between the magnet and the reed switch is increased until the switch opens, thus activating a telemetry oscillator as shown in Fig. 1 [6]. This system operated on the assumptions that the musculature of the bladder is elastic and behaves like a balloon being filled. However, healthy urinary bladder muscle tissue is not flaccid under normal conditions and patients with a sensory paralytic bladder have chronic overdistension and flaccid bladders [7]. Furthermore, both of these methods require surgical intervention and are accompanied by postsurgical pain, long recovery time, and additional costs of healthcare.

Several researchers have implemented various ultrasonic systems and geometrical formulas to quantify the volume of residual urine [7-12]. Although urine volume measurements were relatively inaccurate, the presence or absence of urine in the bladder could be detected. Rise et al. [7] correlated some of these inaccuracies to the variability in bladder contour between individuals, different measurement time intervals, and differences in fecal content in the colon among subjects. Although ultrasonic bladder volume/pressure measurement is a noninvasive procedure, Ulrich reported that the long-term exposure of the body to high ultrasonic intensity greater than $100 \mathrm{~mW} / \mathrm{cm}^{2}$ could pose serious health risks to the body [13].

Several research groups have developed strain gauge sensors to measure bladder volume [14-16]. These sensors can be placed inside or outside of the bladder; they undergo a conformational change as the bladder shape changes. However, the approach of invasively wrapping the sensor around a bladder, as shown in Fig. 1, could not be easily implemented and some strain-gauge sensors gave a low sensing range of $2 \%$, which would not be sufficient for detecting bladder volume changes $[14,15]$. A wireless bladder volume monitoring system using a flexible capacitive-based strain sensor was presented to monitor bladder urine volume with respect to changes in the sensor capacitance [16]. Even though this capacitive-based strain sensor is invasive, its interdigitated finger structure allows it to detect small changes in volume in the urinary bladder far better than the formerly designed strain-gauge sensors.

\section{DESIGN CHALLENGES OF IMPLANTABLE BLADDER SENSORS}

\section{Hermeticity and Biocompatibility}

The durability of an implantable bladder sensor is a very im- portant factor of the sensor design because human urine presents a hostile and corrosive environment to bladder sensors [17]. Biocompatibility of sensor packaging material becomes a challenging problem in such biological environments. To increase the durability of the implantable sensor as well as reduce the risk of infections that the implanted sensor could cause to the human body, several considerations must be made concerning the material of choice for hermetic and biocompatible packaging. Candidate materials that exhibit low cytotoxicity, sufficient immuno-inertness, and biocompatibility include diverse materials such as metals (e.g., nitinol, titanium, stainless steel, and platinum), polymers (e.g., liquid crystal polymers, parylene, polydimethylsiloxane [PDMS], and silicone rubber), various inorganics and ceramics (e.g., zirconia and alumina) [18]. Many miniaturized microfabricated sensors using microelectromechanical systems (MEMS) technology use bio-incompatible materials such as $\mathrm{Si}$, poly-Si, $\mathrm{SiO}_{2}, \mathrm{Si}_{3} \mathrm{~N}_{4}, \mathrm{SiC}$, and $\mathrm{SU}-8$ epoxy photoresists [19]. Since these materials are unavoidable in the MEMS sensor design, a biocompatible encapsulant is essential for implantable MEMS sensors.

A hermetic package is required to prevent body fluids from leaking into the implantable bladder sensor. With a hermetic package, issues such as open circuits, leakage current, electrical shorts due to moisture-promoted dendritic growth, and disconnection of solder-attached components resulting from solder oxidization due to ingression of active gases such as oxygen, could be prevented. Packaging processes are particularly challenging issues associated with MEMS sensor designs because the sensor sensitivity is affected by its packaging [20,21]. MEMS technology employs two materials as substrates for their sensor fabrication: silicon and glass. Corning 7740 (PYREX, Corning, New York, NY, USA), Corning 7070, Corning 1729, Corning 9626, Schott 8330 (Tempax, SCHOTT AG, Mainz, Germany), and SD-2 (HOYA, Tokyo, Japan) are the most common glass materials used in MEMS technology because they have thermal expansion properties close to that of silicon [22,23]. Anodic bonding is the standard technique used for the creation of a hermetic seal between glass and silicon. Currently, most silicon microsensors are made using this technology. It does not require interlayer materials, which simplifies the manufacturing process and eliminates biocompatibility concerns [19].

\section{Drift}

To achieve reliable long-term measurements, an implanted bladder sensor should possess a stable, consistent response over 
its lifetime. Ideally, a change in the response of the sensor should only occur as a result of a change in the quantity to be measured. In our case, that should be a change in urine pressure or volume inside the bladder. However, that is not always the situation in practice because the response of the sensor is also affected by other factors. Therefore, a change in the response of a sensor which is inconsistent with the rate of change of the quantity to be measured is known as drift. In the case of catheter-based sensors, drift is controlled by frequent recalibration through an external reference point. On the other hand, recalibration is impossible for implantable bladder sensors without surgical intervention. Signal drift can be classified into two forms: offset drift and sensitivity drift. Offset drift occurs when the baseline measurement drifts to obscure the desired measurement or in other words, the sensor calibration changes. Sensitivity drift may be attributed to degradation of sensor performance due to material aging and mechanical fatigue resulting from attachment of cells or diaphragm material corrosion. Zhou et al. [24] presented that silicon wafers showed signs of corrosion in bicarbonate buffered saline at $37^{\circ} \mathrm{C}$ after about 6 months. The human urinary bladder environment is dynamic; that is to say, many unknown and parasitic capacitances that could have a significant influence on the whole sensor system exist. For instance, an implantable bladder sensor that incorporates the appropriate materials and operating principles could operate perfectly in vitro, but if a design consideration is not made in terms of its sensing environment (in vivo), like the wet milieu of the urinary bladder, drift and inaccuracy could arise from change in $\mathrm{pH}$, fluid absorption of sensor substrate, biofouling of the sensor, etc. Therefore, it is important to integrate the implanted bladder sensor with drift compensation circuits in order to deliver a reliable and continuous measurement.

\section{Telemetry}

Wireless communication is a promising technology for transmitting/receiving data from the implantable device to an external device without the use of wires. It creates more comfort to the patient while at the same time avoiding complications created by wires. Wireless communication is generally accomplished through the use of radio frequency (RF), optical, sound, or infrared media, with RF being the most common. The medical device community has allocated specific bands for wireless communication of implantable devices. They include: the very high frequency band at $174-216 \mathrm{MHz}$, the ultrahigh frequency bands at $401-406 \mathrm{MHz}$ and $450-470 \mathrm{MHz}$, and several narrow bands within the industrial, scientific and medical bands of $6.765 \mathrm{MHz}$ to $245 \mathrm{GHz}[25,26]$. For an implantable bladder sensor that uses RF communication, a frequency in the low$\mathrm{MHz}$ region provides a good compromise with respect to bandwidth and tissue absorption [27-29]. The appropriate frequency and bandwidth chosen to transmit and receive data from the bladder sensor is chosen based on the location of the implantable device.

Generally, wireless communication takes two forms: active telemetry and passive telemetry. The former requires active electronics such as amplifiers and microcontrollers for wireless communication. Therefore, a power source in the form of an onboard battery or inductive coupling becomes necessary $[30,31]$. The latter does not require power to function and is usually driven by an external device, specifically, an inductive coil. For long-term application of active telemetry, a long lasting power source becomes a necessity and may require a rechargeable battery. Passive telemetry is usually preferred because of the simplicity of its circuitry as well as the smaller size of its design. In both cases, communication distance is a challenging issue because RF quickly dissipates in tissue and bone whereas inductive coupling requires alignment and proper positioning of both internal and external coils for efficient power transfer. Several researchers have presented methods to improve the coupling efficiency of the external coil and implantable sensor coil, regardless of position $[27,32,33]$.

\section{Low Power Consumption and High Data Sampling Rate}

One challenging issue for implantable bladder sensors is the need for low power consumption of the implanted sensor in combination with a high data transmission/reception sampling rate. Many times, a high sampling rate is desired because it provides more information about the measurement quantity. However, a high sampling rate means more power consumption and a shorter battery life. A solution to this issue is an inductively powered bladder sensor receiving power from an external power transfer system, although there is a loss of power while transferring power between the external system and the bladder sensor. Additionally, the rate of the coupling efficiency and the distance between the transceiver and receiver systems should be minimized to obtain a maximum coupling efficiency. Apart from the type of power source used, more power consumption in the implantable bladder sensor leads to dissipation of heat from the sensor that could cause damage to surrounding tissues. As a result of this, International Standard Organiza- 
tion 14708 , clause 17 , clearly states that no implantable device surface temperature shall exceed $2^{\circ} \mathrm{C}$ above body temperature [20]. Thanks to the rapid advancement in microfabrication and micromachining, most implantable bladder sensors are designed to have a very small size and consume very little power.

\section{Compatibility Issues With Popular Imaging Tools}

Another problem that implantable bladder sensors encounter is their poor compatibility with current imaging devices such as CT, MRI, and ultrasound. Soft tissue imaging such as neurological imaging, oncological imaging, and muscular imaging, as well as many others necessitate the use of MRI. The use of ferrites and metallic packaging in implantable bladder sensors to effectively improve wireless communication and hermeticity, respectively, could interfere with MRI and cause heat dissipation, image distortion, and leakage current due electromagnetic induction. Furthermore, high doses of x-rays employed in CT scans could erase important data/information from memory chips and other data storage devices in the implantable bladder sensor. Moreover, the use of high ultrasonic energy for imaging could cause disruption in the electronic circuitry of the implantable bladder sensor, possibly leading to device failure, packaging issues or potential danger to the patient [20]. Therefore, implantable bladder sensors with new and safe technologies that would allow MRI compatibility and low-dose based xray acquisition should be implemented.

\section{IMPROVEMENTS/EMERGING TRENDS IN IMPLANTABLE BLADDER SENSOR DESIGN}

\section{Hermeticity and Biocompatibility}

Polymers such as silicone [34] or liquid crystal polymers [35] tend to absorb moisture during long-term use within the body. Therefore, proper caution must be exercised when using such polymers in the bladder because they could cause significant drift or leaks within the sealed cavities of your design. Apart from the materials used for the circuitry and sensor element, the interconnect materials such as lead, gold-tin solders, and epoxies should be encapsulated to produce a hermetic implantable sensor [33]. Hermetic packaging is essential in preventing failure of the implanted active circuitry as well as preventing lethal substances from leaching into the bladder. From time to time, the stress induced by the hermetic packaging process interferes with the sensitivity of a pressure sensitive membrane. In such cases, the pressure sensitive membrane is created within the casing. Any pressure exerted on the deflectable casing membrane is transduced onto the sensing membrane of the pressure sensor by using an incompressible fluid (e.g., silicone gel or oil) [36,37].

Another way to enhance a hermetic and biocompatible package is to have a second encapsulation, commonly with silicone rubber or a parylene coating [38-41]. Silicone and parylene coatings were found to improve biocompatibility and reduce offset drift of implantable bladder sensors; however, it was discovered that they could also reduce the sensitivity of the implantable bladder sensor [41-45]. Furthermore, PDMS encapsulation was found to have a negative impact on frequency response by degrading it [46]. Drug coating is known to benefit sensor performance by reducing the adsorption of proteins and other cellular material onto the sensing system of an implantable bladder sensor. Furthermore, sodium 2-acrylamido2-methylpropyl sulfonate coating on the silicone encapsulation could prevent salt deposition on the sensor material [38]. Lastly, the use of parylene $\mathrm{C}$ and thin-film platinum could reduce the complexities of hermetic and biocompatible packages [47].

\section{Drift}

A well-known method to eliminate baseline drift is to compare the desired signal to a reference signal and then compensate for the error. For strain gauge pressure sensors, baseline drift coming from an environmental influence could be eliminated by introducing a pressure insensitive capacitive element as a reference to the sensing element in a differential signal circuitry $[48,49]$. Another drift compensation method is to implement a differential pressure sensing technique that uses another sensor, outside of the bladder, to measure the difference in pressure between the inside and outside of the bladder wall. This technique could be a way to moderately improve baseline drift [50]. In addition, averaging the output data of a sensor array could compensate for the drift.

To compensate for the temperature drift, additional circuitry or temperature insensitive materials have been used in the bladder sensors. The introduction of an on-board temperature compensation circuit [51-53] or external signal processing circuitry [48,54] could help in the recalibration of the sensor response in vivo. Temperature-insensitive materials such as temperature-insensitive quartz should be used for sensor transducers as a way to minimize temperature drift $[53,55]$.

To compensate for the biofouling drift, ultrasonic energy could be used to remove biofouling by vibrating the sensor 
membrane (e.g., piezoelectric membrane transducer) at ultrasonic frequencies while ensuring the ultrasonic intensity is not high enough to cause any damage [56]. Coating the bladder sensor with silicone or parylene to prevent biofouling and immune system response could also minimize sensitivity drift.

\section{Telemetry and Power Transfer}

Long-term, continuous power transfer and efficient power transfer between inductively coupled coils are the two major issues commonly associated with telemetry and power transfer. Currently, power is transferred to the circuitry of the implantable bladder sensor by the use of a battery-based approach, having a limited lifetime, or an RF-based approach, having limited coupling efficiency.

For short-term monitoring of bladder volume/pressure, battery-based implantable sensors are suitable. Adapting power conservation mechanisms such as device hibernation after 5-10 minutes without usage could be implemented to enhance the battery life and operational efficiency. For long-term monitoring of bladder pressure, an RF-based inductive coupling approach can be used [57]. However, the coupling efficiency be- tween the implantable bladder sensor coil and external coil should be taken into consideration since the external coil/transmitter must be continuously worn to provide power to the internal bladder sensor if the coupling efficiency is low. To circumvent this issue, a hybrid system was created that combines the battery and RF charging components $[32,33,58]$. A hybrid system could eliminate the need for continuous wearing of a bulky external coil by allowing the RF-based rechargeable battery to operate the sensor during the day, while an external coil recharges it at night. In a setting whereby the patient is constantly moving (e.g., neonates), inductive coupling may not be very efficient for data transmission and/or reception.

Currently, several researchers are focused on enhancing the transmission of power to the implantable bladder sensor, by improving or circumventing coupling issues between the two coils, as well as developing new ways to reduce attenuation-related effects in vivo. Other researchers have developed an adaptive power control mechanism to monitor the sufficiency of power delivered to an implantable sensor and its circuitry, and to store this power in a capacitor if the power received is not sufficient $[27,59]$. This technique circumvents poor coupling is-
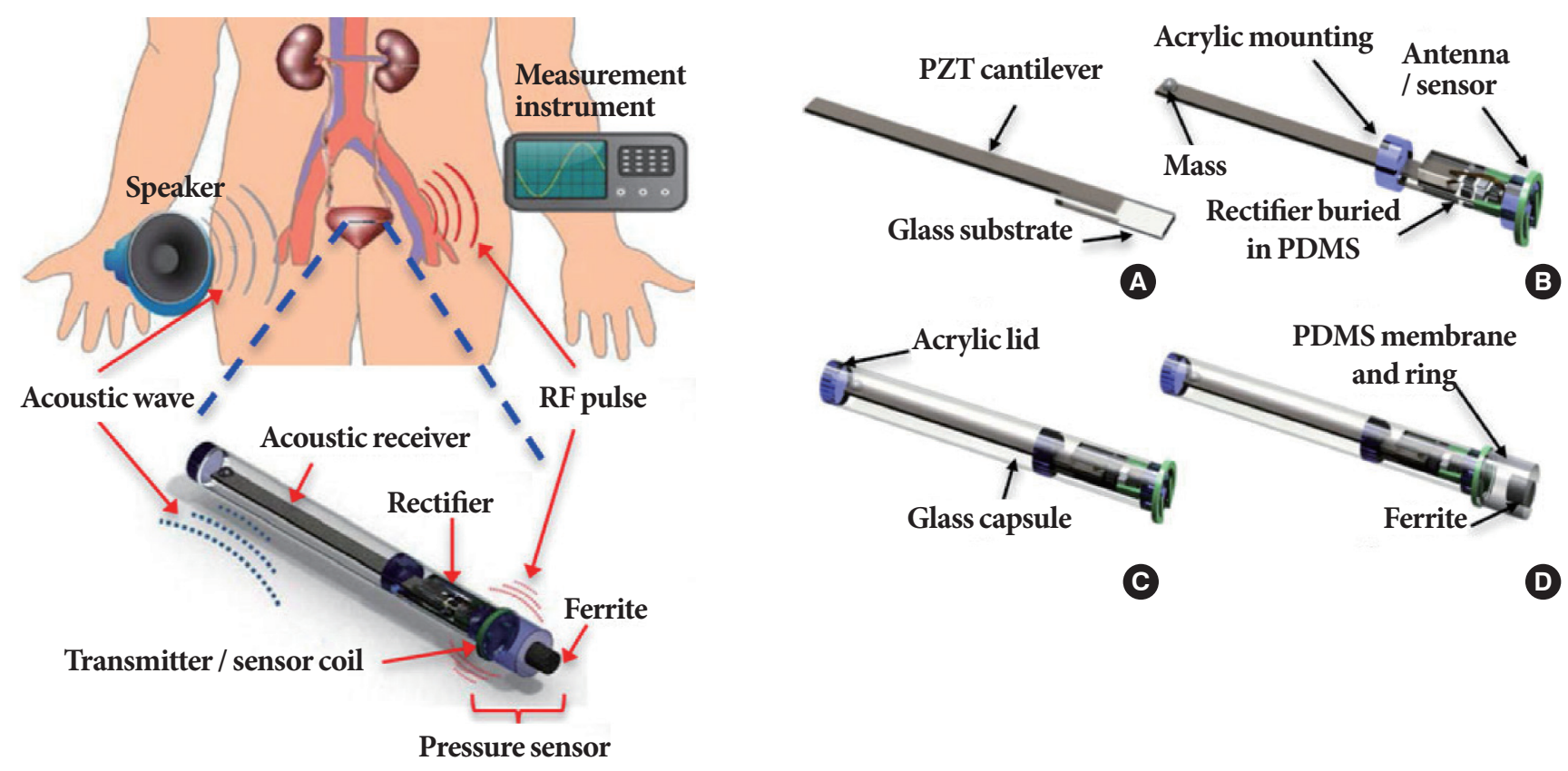

Fig. 2. An implantable bladder pressure sensor using PZT as energy source where (A) an acoustic receiver (PZT cantilever) and rectifier circuitry are assembled on a glass substrate; (B) an acrylic holder, proof of mass, and antenna/sensor coil are added; (C) the assembled parts are inserted into a glass tube and an acrylic lid is attached; and (D) the inductive sensor with PDMS membrane and ferrite core are added. Adapted from Kim A, et al. IEEE Trans Biomed Eng 2014;61:2209-17 [61], with permission of the Institute of Electrical and Electronics Engineers. RF, radio frequency; PZT, piezoelectric lead zirconat titanate; PDMS, polydimethylsiloxane. 
sues and uses smart implantable device technologies to operate when the power becomes sufficient. Other efforts to improve coupling efficiency involve the use of two or more external coils/transmitters within a system. Among the coils, the bestcoupled coil to the implantable bladder sensor coil is selected and the others are turned off [60]. This technique ensures proper coupling between internal and external coils, while at the same time ensuring efficiency of power transfer regardless of the bladder sensor position.

Many inductor-capacitor tank-based implantable bladder sensors encounter variation issues in their dielectric constant once they are implanted into a wet environment such as the inside of the bladder $\left(\varepsilon_{\mathrm{r}} \approx 1\right.$ in air and $\varepsilon_{\mathrm{r}} \approx 80$ for saline solution) [35]. These changes are usually due to the increase in the number of parasitic and other unwanted capacitances that could degrade the resonant frequency as well as the quality factor of the implanted sensor. Silicone encapsulation is known to minimize dielectric constant variations in the bladder but at the cost of reducing the sensitivity of the entire sensor system. An alternative solution to this problem is the use of small ferrite rods within the coils to improve the quality factor of the coils for improved inductive coupling.

Piezoelectric ceramic materials such as lead zirconate titanate (PZTs) could be an alternative means of harvesting energy to power implantable bladder sensors. Kim et al. [61] used an acoustic signal generated by an external speaker to cause an implanted piezoelectric cantilever, connected to a pressure sensor within the bladder, to vibrate at its resonant frequency as shown in Fig. 2. The low frequency of the acoustic signal allowed it to be transported through the body to the PZT with minimal signal loss, with no concerns of orientation or alignment related effects. This particular technology eliminates the alignment and distance issues associated with RF-based telemetry and simplifies implanted sensor design by removing the need for a sophisticated onboard receiver system.

\section{CONCLUSIONS}

From the patient's point of view, catheterization and catheterbased sensors reduce their quality of life after surgical intervention and can cause infections during prolonged use. Currently available implantable bladder sensors may be more comfortable for the patient; however, they have limitations in accuracy due to drift, biocompatibility, hermetic packaging, communication, operating power, and incompatibility with popular medical im- aging tools. To minimize drift, improve hermeticity, and enhance biocompatibility of implantable bladder sensors, adaptations using temperature-insensitive deflectable diaphragms and biocompatible encapsulation with silicone, PDMS, or parylene are being explored. To improve power issues of implantable bladder sensors, a hybrid system that uses a rechargeable battery and an external power transfer system could be utilized. Moreover, power-harvesting materials such as PZTs could be an alternative means to power implantable bladder sensors.

Thus, several techniques and the status of current technologies in either solving or circumventing these limitations and issues were reviewed. If the above methods are implemented, implantable bladder sensors will not only operate in a manner as good as catheter-based sensors, but will also improve the patient's quality of life.

\section{REFERENCES}

1. Coyne KS, Wein A, Nicholson S, Kvasz M, Chen CI, Milsom I. Economic burden of urgency urinary incontinence in the United States: a systematic review. J Manag Care Pharm 2014;20:130-40.

2. Ramesh MV, Raj D, Sanjeevan K, Dilraj N. Design of wireless real time artificial sphincter control system for urinary incontinence. In: 2014 International Symposium on Technology Management and Emerging Technologies (ISTMET 2014); 2014 May 27-29; Bandung, Indonesia. Piscataway (NJ): IEEE; 2014.

3. Yu L, Kim BJ, Meng E. Chronically implanted pressure sensors: challenges and state of the field. Sensors (Basel) 2014;14:20620-44.

4. Mendez A, Sawan M, Minagawa T, Wyndaele JJ. Estimation of bladder volume from afferent neural activity. IEEE Trans Neural Syst Rehabil Eng 2013;21:704-15.

5. Waltz FM, Timm GW, Bradley WE. Bladder volume sensing by resistance measurement. IEEE Trans Biomed Eng 1971;18:42-6.

6. Dreher RD, Timm GW, Bradley WE. Bladder volume sensing by local distension measurement. IEEE Trans Biomed Eng 1972;19:2478.

7. Rise MT, Bradley WE, Frohrib DA. An ultrasonic bladder-volume sensor. IEEE Trans Biomed Eng 1979;26:709-11.

8. Holmes J. Ultrasonic studies of bladder filling and contour. In: Hinman F, editor. Hydrodynamics of mictwition. Springfield: Thomas; 1971. p. 303-17.

9. West KA. Sonocystography: a method for measuring residual urine. Scan J Urol Nephrol 1967;1:68-70.

10. Doust BD, Baum JK, Maklad NF, Baum RF. Determination of organ volume by means of ultrasonic B-mode scanning. J Clin Ultra- 
sound 1974;2:127-30.

11. Pedersen JF, Bartrum RJ, Grytter C. Residual urine determination by ultrasonic scanning. Am J Roentgenol Radium Ther Nucl Med 1975;125:474-8.

12. McLean GK, Edell SL. Determination of bladder volumes by gray scale ultrasonography. Radiology 1978;128:181-2.

13. Ulrich WD. Ultrasound dosage for nontherapeutic use on human beings: extrapolations from a literature survey. IEEE Trans Biomed Eng 1974;21:48-51.

14. Rajagopalan S, Sawan M, Ghafar-Zadeh E, Savadogo O, Chodavarapu P. A polypyrrole-based strain sensor dedicated to measure bladder volume in patients with urinary dysfunction. Sensors 2008;8:5081-95.

15. Gutierrez CA, Meng E. Low-cost carbon thick-film strain sensors for implantable applications. J Micromech Microeng 2010;20: 095028.

16. Hung C, Uday T, Vaibhav L, Ai-Ling L, Yuan-Bo P, Chiao J. A wireless bladder volume monitoring system using a flexible capacitance-based sensor. In: 2013 IEEE topical conference on biomedical wireless technologies, networks, and sensing systems (BioWireless); 2013 Jan 20-23; Austin (TX), USA. Piscataway (NJ): IEEE; 2013.

17. Williams DF. Corrosion of implant materials. Ann Rev Mater Sci 1976;6:237-66.

18. Yuen T, Agnew W, Bullara L, McCreery DB. Biocompatibility of electrodes and materials in the centra lnervous system. In: Agnew W, McCreery DB, editors. Neural prostheses: fundamental studies. Englewood Cliffs (NJ): Prentice Hall; 1990, p. 171-321.

19. Kotzar G, Freas M, Abel P, Fleischman A, Roy S, Zorman C, et al. Evaluation of MEMS materials of construction for implantable medical devices. Biomaterials 2002;23:2737-50.

20. Jiang $G$. Design challenges of implantable pressure monitoring system. Front Neurosci 2010;4:29.

21. Joung YH. Development of implantable medical devices: from an engineering perspective. Int Neurourol J 2013;17:98-106.

22. Rogers T, Kowal J. Selection of glass, anodic bonding conditions and material compatibility for silicon-glass capacitive sensors. Sens Actuator A 1995;46:113-20.

23. Harz M, Engelke H. Curvature changing or flatening of anodically bonded silicon and borosilicate glass. Sens Actuator A 1996;55:2019.

24. Zhou D, Mech B, Greenberg R. Accelerated corrosion tests on Silicon wafers for implantable medical devices [abstract 363]. In: Proceedings of 198th Electrochemical Society Meeting; 2000 Oct 2227; Phoenix (AZ), USA. Pennington (NJ): Electrochemical Society;
2000 .

25. Akyildiz IF, Su W, Sankarasubramaniam Y, Cayirci E. Wireless sensor networks: a survey. Computer Netw 2002;38:393-422.

26. Budinger TF. Biomonitoring with wireless communications. Annu Rev Biomed Eng 2003;5:383-412.

27. Poon AS, O'Driscoll S, Meng TH. Optimal frequency for wireless power transmission into dispersive tissue. IEEE Trans Antenna Propag 2010;58:1739-50.

28. DeHennis AD. Remotely-powered wireless monitoring systems [dissertation]. Ann Arbor (MI): University of Michigan; 2004.

29. Yu H. A wireless microsystem for multichannel neural recording microprobes [dissertation]. Ann Arbor (MI): University of Michigan; 2004.

30. Abouei J, Brown JD, Plataniotis KN, Pasupathy S. Energy efficiency and reliability in wireless biomedical implant systems. IEEE Trans Inf Technol Biomed 2011;15:456-66.

31. Puers R. Linking sensors with telemetry: impact on the system design. Sens Actuator A 1996;52:169-74.

32. Suster M, Young D. Wireless recharging of battery over large distance for implantable bladder pressure chronic monitoring. In: Proceedings of the 16th International on Solid-State Sensors, Actuators and Microsystems Conference (TRANSDUCERS); 2011 Jun 5-9; Beijing, China. Piscataway (NJ): IEEE; 2011.

33. Chow EY, Chlebowski AL, Chakraborty S, Chappell WJ, Irazoqui PP. Fully wireless implantable cardiovascular pressure monitor integrated with a medical stent. IEEE Trans. Biomed. Eng. 2010;57: 1487-96.

34. Kim J, Lee H, Cha S, Choi B. Fabrication and evaluation of implantable pressure sensor using strain gauge. In: Proceedings of the Biomedical Engineering International Conference (BMEiCON); 2012 Dec 5-7; Ubon Ratchathani, Thailand. Piscataway (NJ): IEEE; 2012.

35. Fonseca MA, Allen MG, Kroh J, White J. Flexible wireless passive pressure sensors for biomedical applications. In: Proceedings of the Solid-State Sensor, Actuator, and Microsystems Workshop; 2006 Jun 4-8; Hilton Head Island (SC), USA. Ann Arbor (MI): Wireless Integrated MicroSensing \& Systems; 2006.

36. Cong P, Ko WH, Young DJ. Wireless batteryless implantable blood pressure monitoring microsystem for small laboratory animals. IEEE Sens J 2010;10:243-54.

37. Majerus SJ, Fletter PC, Damaser MS, Garverick SL. Low-power wireless micromanometer system for acute and chronic bladderpressure monitoring. IEEE Trans Biomed Eng 2011;58:763-7.

38. Axisa F, Jourand P, Lippens E, Rymarczyk-Machal M, De Smet N, Schacht E, et al. Design and fabrication of a low cost implantable 
bladder pressure monitor. Conf Proc IEEE Eng Med Biol Soc 2009;4864-7.

39. Chen PJ, Rodger DC, Saati S, Humayun MS, Tai YC. Microfabricated implantable Parylene-based wireless passive intraocular pressure sensors. J Microelectromec Syst 2008;17:1342-51.

40. Siwapornsathain E, Lal A, Binard J. A telemetry and sensor platform for ambulatory urodynamics. In: Proceedings of the Biology 2nd Annual International IEEE-EMB Special Topic Conference on Microtechnologies in Medicine, Madison, WI, USA, 2002;283-7.

41. Takahata K, DeHennis A, Wise KD, Gianchandani YB. A wireless microsensor for monitoring flow and pressure in a blood vessel utilizing a dual-inductor antenna stent and two pressure sensors. In: Proceedings of the 17th IEEE International Conference on Micro ElectroMechanical Systems; 2004 Jany 25-29; Maastricht, The Netherlands. Piscataway (NJ): IEEE; 2004.

42. Chen PJ, Rodger DC, Agrawal R, Saati S, Meng E, Varma R, et al. Implantable micromechanical parylene-based pressure sensors for unpowered intraocular pressure sensing. J Micromech Microeng 2007;17:1931-8.

43. Aquilina K, Thoresen M, Chakkarapani E, Pople I, Coakham HB, Edwards RJ. Preliminary evaluation of a novel intraparenchymal capacitive intracranial pressure monitor laboratory investigation. J Neurosurg 2011;115:561-9.

44. Fan Z, Engel JM, Chen J, Liu C. Parylene surface-micromachined membranes for sensor applications. J Microelectromec Syst 2004; 13:484-90.

45. Jourand P, Puers R. The BladderPill: an in-body system logging bladder pressure. Sens Actuator A 2010;162:160-6.

46. Schnakenberg U, Kruger C, Pfeffer JG, Mokwa W, vom Bogel G, Gunther R, et al. Intravascular pressure monitoring system. Sens Actuator A 2004;110:61-7.

47. Gutierrez C, Meng E. A subnanowatt microbubble pressure sensor based on electrochemical impedance transduction in a flexible allparylene/parylene package. In: Proceedings of the 24th International Conference on Micro Electro Mechanical Systems; 2011 Jan 23-27; Cancun, Mexico. Piscataway (NJ): IEEE; 2011.

48. Cleven NJ, Muntjes JA, Fassbender H, Urban U, Gortz M, Vogt H, et al. A novel fully implantable wireless sensor system for monitoring hypertension patients. IEEE Trans Biomed Eng 2012;59:312430.

49. Hierold C, Clasbrumme B, Behrend D, Scheiter T, Steger M, Oppermann $\mathrm{K}$, et al. Implantable low power integrated pressure sensor system for minimal invasive telemetric patient monitoring. In: Proceedings of the the 11th Annual International Workshop on Micro Electro Mechanical Systems; 1998 Jan 25-29; Heidelberg,
Germany. Piscataway (NJ): IEEE; 1998.

50. Tan R, McClure T, Lin CK, Jea D, Dabiri F, Massey T, et al. Development of a fully implantable wireless pressure monitoring system. Biomed Microdevices 2009;11:259-64.

51. Koley G, Liu J, Nomani MW, Yim M, Wen X, Hsia TY. Miniaturized implantable pressure and oxygen sensors based on polydimethylsiloxane thin films. Mater Sci Eng R Rep 2009;29:685-90.

52. Majerus SJ, Garverick SL, Suster MA, Fletter PC, Damaser MS. Wireless, ultra-low-power implantable sensor for chronic bladder pressure monitoring. ACM J Emerg Technol Comput Syst 2012;8. Article No. 11.

53. Liang B, Fang L, Tu C, Zhou C, Wang X, Wang Q, et al. A novel implantable saw sensor for blood pressure monitoring. In: Proceedings of the 16th International on Solid-State Sensors, Actuators and Microsystems Conference (TRANSDUCERS); 2011 Jun 5-9; Beijing, China. Piscataway (NJ): IEEE; 2011.

54. Chua-Chin Wang, Chi-Chun Huang, Jian-Sing Liou, Yan-Jhin Ciou, I-Yu Huang, Chih-Peng Li, et al. A Mini-Invasive Long-Term Bladder Urine Pressure Measurement ASIC and System. IEEE Trans Biomed Circuits Syst 2008;2:44-9.

55. Li C, Wu PM, Shutter LA, Narayan RK. Dual-mode operation of flexible piezoelectric polymer diaphragm for intracranial pressure measurement. Appl Phys Lett 2010;96:053502.

56. Bott TR. Biofouling control with ultrasound. Heat Transf Eng 2000;21:43-9.

57. Frischholz M, Sarmento L, Wenzel M, Aquilina K, Edwards R, Coakham HB. Telemetric implantable pressure sensor for shortand long-term monitoring of intracranial pressure. Conf Proc IEEE Eng Med Biol Soc 2007;514.

58. Fletter PC, Majerus S, Cong P, Damaser MS, Ko W, Young DJ, et al. Wireless micromanometer system for chronic bladder pressure monitoring. In: Proceedings of the 2009 Sixth International Conference on Networked Sensing Systems (INSS); 2009 Jun 17-19; Pittsburgh (PA), USA. Piscataway (NJ): IEEE; 2011.

59. Kilinc EG, Moya AC, van Lintel H, Renaud P, Maloberti F, Wang Q, et al. Remotely powered implantable heart monitoring system for freely moving animals. In: Proceedings of the 2013 5th IEEE International Workshop on Advances in Sensors and Interfaces (IWASI); 2013 Jun 13-14; Bari, Italy. Piscataway (NJ): IEEE; 2011.

60. Coosemans J, Puers R. An autonomous bladder pressure monitoring system. Sens Actuator A 2005;123-124:155-61.

61. Kim A, Powell CR, Ziaie B. An implantable pressure sensing system with electromechanical interrogation scheme. IEEE Trans Biomed Eng 2014;61:2209-17. 